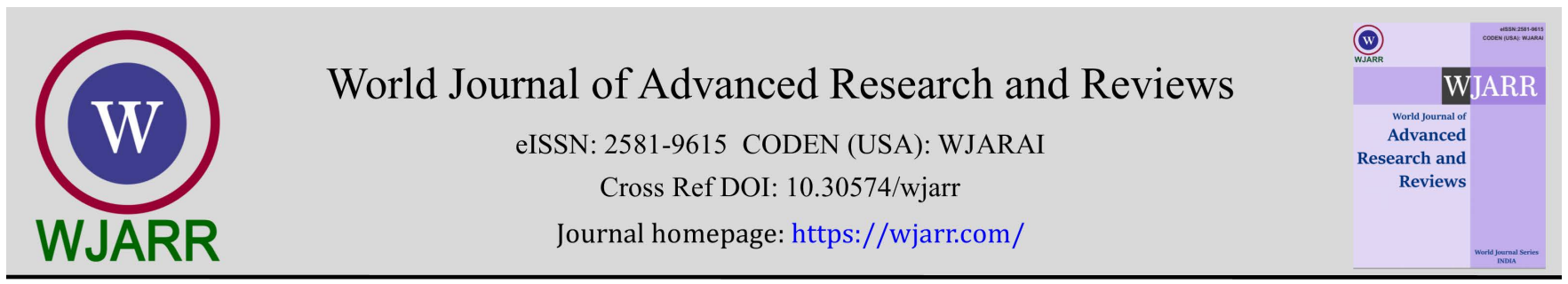

(CASE REPORT)

\title{
Rhabdomyosarcoma of the prostate in children: A Case Report
}

Kanta Ka 1, ${ }^{*}$, Lissoune Cissé ${ }^{3}$, Oumar Gaye ${ }^{4}$, Babacar Sine 2, 5, Mohamed Jalloh 4,5, Ndeye Aby Ndoye 3,5, Gabriel Ngom ${ }^{3,5}$, Alain Khassimou Ndoye 2,5 and Papa Macoumba Gaye 1,5

${ }^{1}$ Radiotherapy Department of the National Universitary Hospital Center Dalal Jamm, Guédiawaye, Senegal.

${ }^{2}$ Urology Department of the Hospital Universitary Center Aristide Le Dantec, Dakar, Senegal.

${ }^{3}$ Department of Pediatric Surgery, Albert Royer Children's Hospital, Dakar, Senegal.

${ }^{4}$ Urology Department, General Hospital Idrissa Pouye, Grand Yoff, Senegal.

${ }^{5}$ Cheikh Anta Diop University of Dakar, Senegal.

World Journal of Advanced Research and Reviews, 2021, 11(03), 312-315

Publication history: Received on 16 August 2021; revised on 25 September 2021; accepted on 27 September 2021

Article DOI: https://doi.org/10.30574/wjarr.2021.11.3.0475

\begin{abstract}
Context: The therapeutic transition from mutilating surgery to external radiotherapy followed by brachytherapy is increasingly effective in the treatment of rhabdomyosarcoma.

Case presentation: 13-year-old child with no medical and surgical history, received for complete urine retention. Imaging examinations revealed a prostatic mass of $6 \mathrm{~cm}$. The anatomopathological examination reveals an embryonic rhabdomyosarcoma. There was no recurrence after 3 years of follow-up after a multimodal treatment combining chemotherapy, external radiotherapy and high dose interstitial brachytherapy.
\end{abstract}

Conclusion: Vesicoprostatic rhabdomyosarcomas in children are rare. The therapeutic strategy has evolved over the years owing to the technological advances in external radiotherapy and brachytherapy.

Keywords: Rhabdomyosarcoma; Children; Brachytherapy; Prostate; Cancer

\section{Introduction}

Rhabdomyosarcomas are the most common type in childhood genitourinary tract sarcomas with the embryonal subtype mainly in the bladder and prostate [1]. Multimodal conservation therapy without surgery shows good survival in multiinstitutional studies [2,3]. We report a case followed and treated without surgery in our Department.

\section{Case presentation}

This is a 13-year-old child, with no known history, received for complete urine retention. CT scan (CT) and magnetic resonance imaging (MRI) revealed a $6 \mathrm{~cm}$ prostate mass, without adenopathy or distant abnormalities. Transrectal biopsy of the prostate followed by pathological examination revealed an embryonal rhabdomyosarcoma. PET-CT showed a non-homogeneous enlargement of the prostate with diffuse and slightly more intense FDG uptake in the right part. Bone marrow biopsy showed no bone marrow invasion. The patient was treated according to the RMS2005 protocol of subgroup E. He received three well-tolerated courses of VIA (ifosfamide, vincristine, actinomycin). The evaluation MRI showed a partial response of 30\%. Three courses of chemotherapy were added, with good tolerance. External radiotherapy with a dose of $41.4 \mathrm{~Gy}$ at a rate of $1.8 \mathrm{~Gy}$ was then performed, followed by a well-tolerated high

\footnotetext{
${ }^{*}$ Corresponding author: Kanta Ka

Radiotherapy Department of the National Universitary Hospital Center Dalal Jamm, Guédiawaye, Senegal. 
dose rate interstitial brachytherapy. Clinical and radiological examinations (MRI, PET) at 3 years follow-up showed no recurrence.
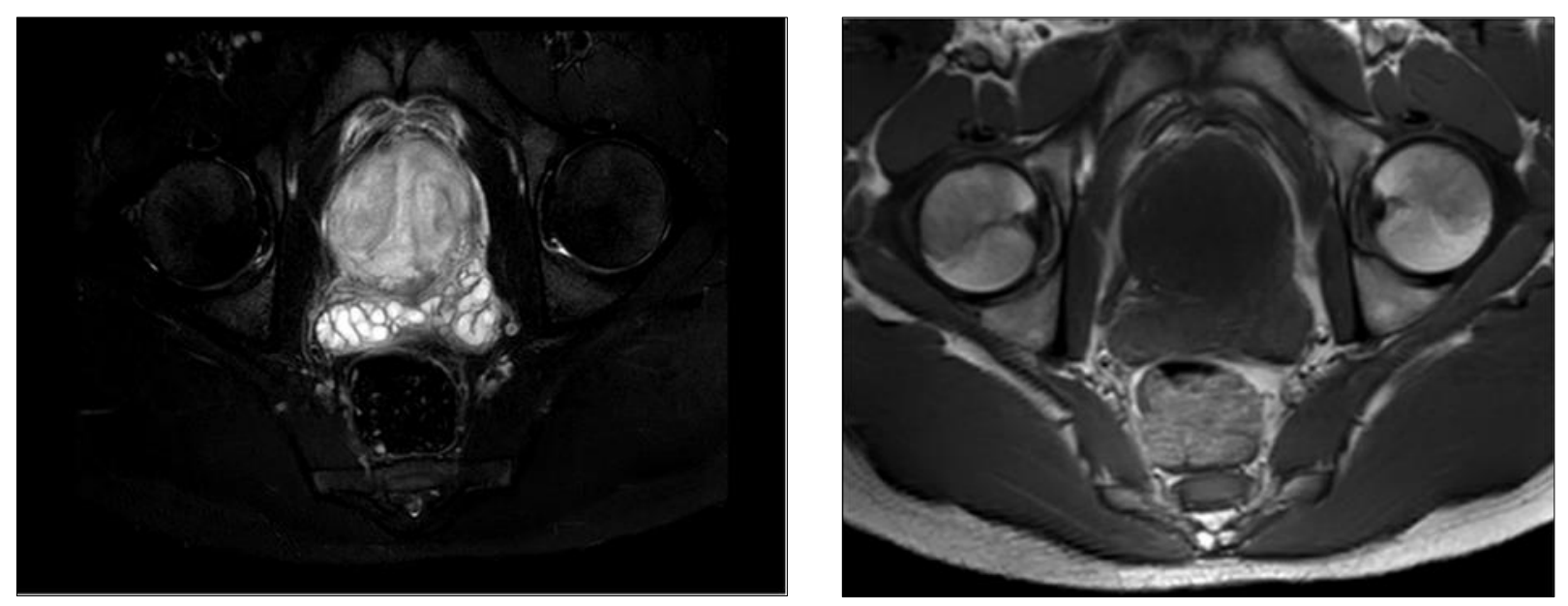

Figure 1 Pre-therapy T2/T1 axial MRI in a 13-year-old child with vesicoprostatic rhabdomyosarcoma
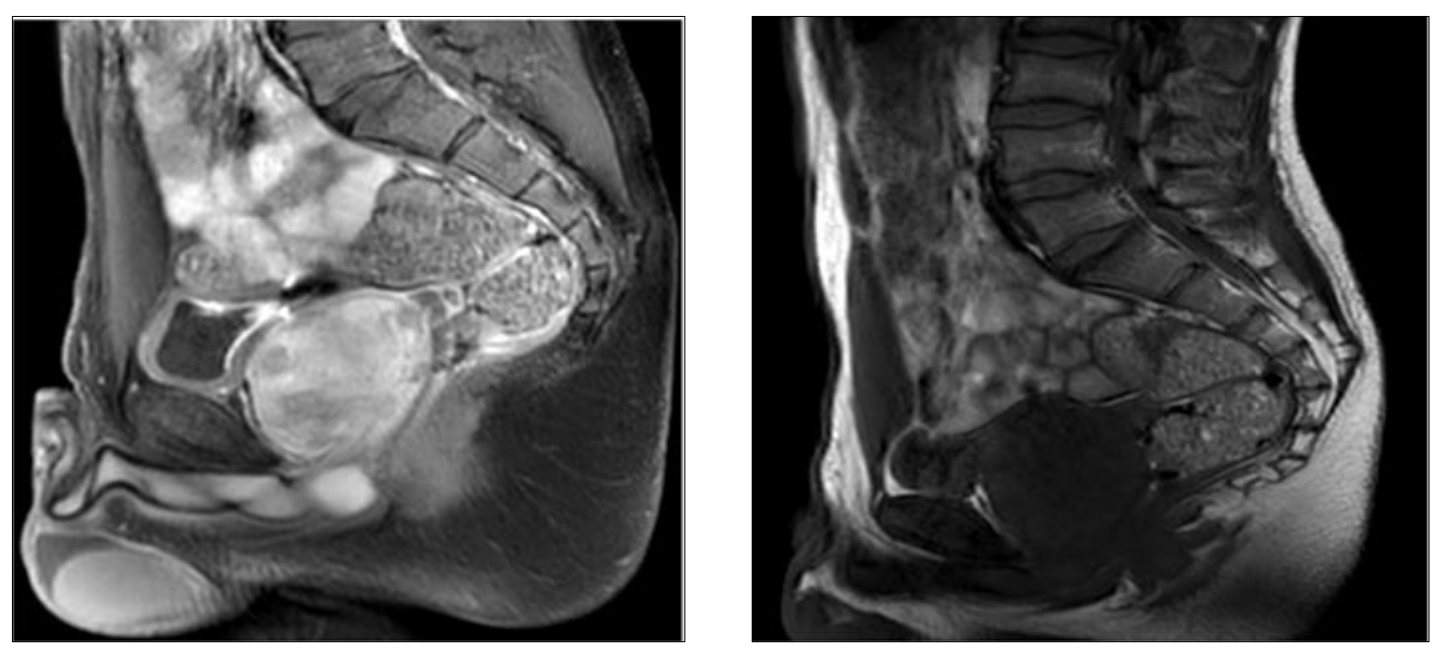

Figure 2 Pre-therapy T2/T1 sagittal MRI in a 13-year-old child with vesicoprostatic rhabdomyosarcoma

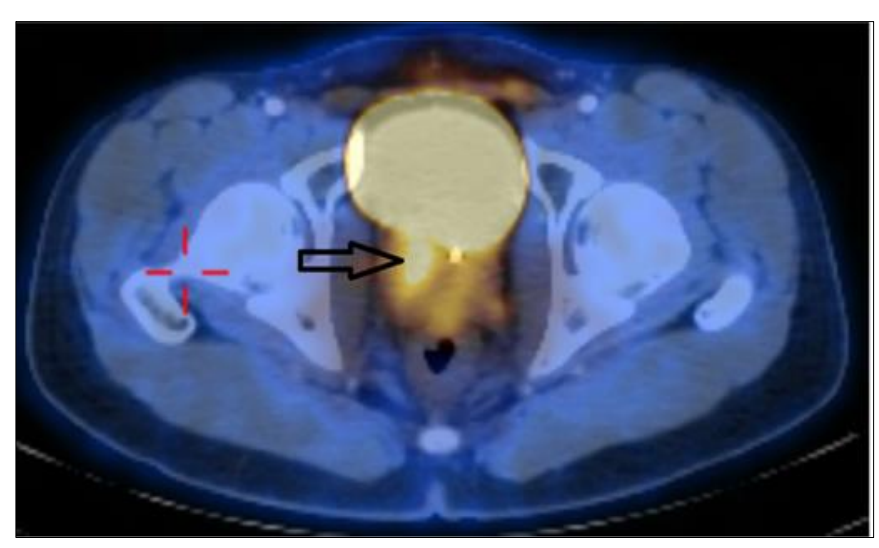

Figure 3 Pre-therapy axial fusion PET in a 13-year-old child with vesicoprostatic rhabdomyosarcoma 

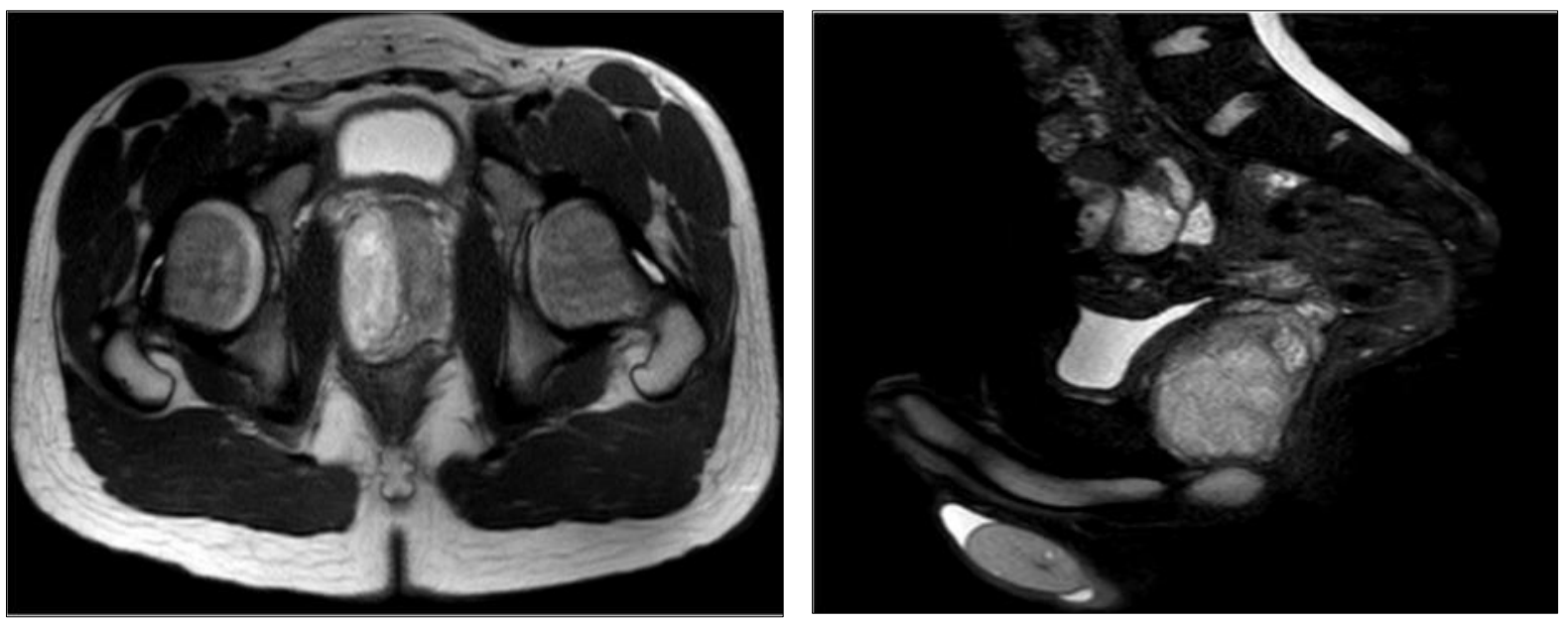

Figure 4 Axial and sagittal T2 MRI surveillance after 3 courses of VIA in a 13-year-old child with vesicoprostatic rhabdomyosarcoma
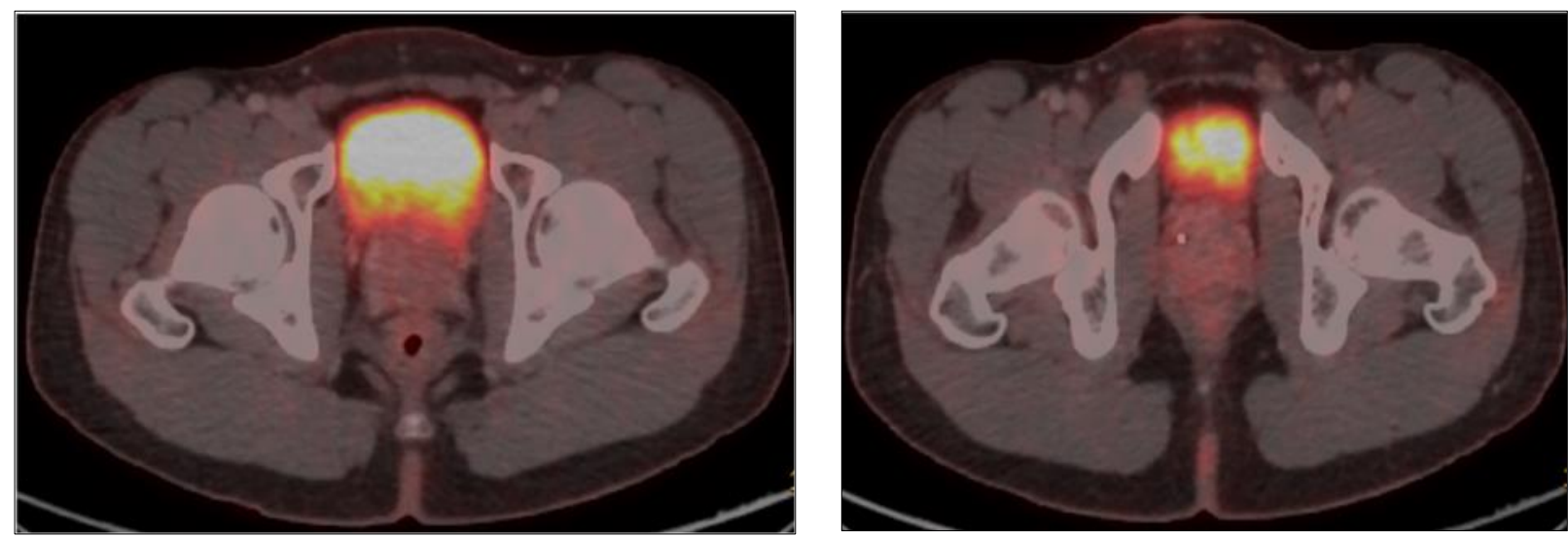

Figure 5 Axial PET after chemotherapy, radiotherapy and brachytherapy in a 13-year-old child with vesicoprostatic rhabdomyosarcoma

\section{Discussion}

Both bladder and prostate localization are the most frequent in pediatric rhabdomyosarcomas [4]. These tumors are characterized by chemosensitivity, making chemotherapy one of their primary treatments. When a tumor remnant remains after chemotherapy, local treatment is required [5]. Historically, radical surgery was the standard treatment for genitourinary rhabdomyosarcoma in children. The current approach consists of organ preservation by chemotherapy followed by external radiotherapy and high dose rate interstitial brachytherapy [6, 7, 8]. These treatments are not without complications, particularly radiotherapy with its late toxicity [8, 9, 10]. Irradiation techniques are undergoing a major evolution, allowing for a considerable reduction in toxicity while at the same time improving therapeutic efficacy. In the context of organ preservation, brachytherapy plays a fundamental role thanks to its ballistic characteristics linked to a high dose gradient at the tumor level with a peripheral dose decay allowing to minimize toxicity to the neighboring organs [5]. Usually the principle of treatment is based on bladder surgery while ensuring that it is complete at the upper bladder limit of the prostate and irradiating the prostate region [5]. Nevertheless, the improved accuracy of external beam radiation therapy with the emergence of proton therapy has made it possible to replace surgery. The results of brachytherapy in rhabdomyosarcoma are mainly published by teams whose children had their treatment at the Gustave Roussy Institute $[11,12]$. The series by Martelli et al showed that out of a total of 26 patients treated by surgery (partial cystectomy or partial prostatectomy) followed by brachytherapy with a 4-year follow-up, 24 children were alive. Of these, 22 were in complete clinical and radiological remission [13]. Our patient has no clinical and/or radiological signs of recurrence after 3 years of follow-up. 
This clinical case shows the efficacy of multimodal treatment and underlines the interest of a multidisciplinary approach especially in pediatric oncology. Indeed, both diagnosis and treatment require expertise in various specialties.

\section{Conclusion}

Vesicoprostatic rhabdomyosarcomas in children are rare. The therapeutic strategy has evolved over the years thanks to technological advances in external radiotherapy and brachytherapy.

\section{Compliance with ethical standards}

\section{Acknowledgments}

We thank the patient for agreeing to participate in this article.

\section{Disclosure of conflict of interest}

The authors declare that they have no conflict of interest.

\section{Statement of informed consent}

Informed consent was obtained from the patient family.

\section{References}

[1] Dasgupta R, Rodeberg DA. Update on rhabdomyosrcome. Semin Pediatr Surg. 2012; 21: 68-78.

[2] Kieran K, Shnorhavorian M. Current standards of care in bladder and prostate rhabdomyosarcoma. Urol Oncol. 2016; 34: 93-102.

[3] Rodeberg DA, Anderson JR, Arndt CA, et al. Comparison of outcomes based on treatment algorithms for rhabdomyosarcoma of the bladder/prostate: combined results from the Children's Oncology Group, German Cooperative Soft Tissue Sarcoma Study, Italian Cooperative Group, and International Society of Pediatric Oncology Malignant Mesenchymal Tumors Committee. Int J Cancer. 2011; 128: 1232-1239.

[4] Haie-Meder C, Martelli H, Dumas I, Mazeron R, Oberlin O. Curiethérapie dans les sarcomes en pédiatrie: techniques, indications, résultats et perspectives. Bull Cancer. 2011; 98: 549-557.

[5] Haie-Meder C, Mazeron R, Martelli H, Oberlin O. Place de la curiethérapie dans les rhabdomyosarcomes pédiatriques. 2013; 17: 155-158.

[6] Harel M, Ferrer FA, Shapiro LH, et al. Future directions in risk stratification and therapy for advanced pediatric genitourinary rhabdomyosarcoma. Urol Oncol. 2016; 34: 103-115.

[7] Komasara L, Stefanowicz J, Bryks-Laszkowska A, et al. Reconstructive option after radical mutilating surgery in children with génito-urinary rhabdomyosarcoma: when sparing the bladder is not an option. Int J Urol. 2016; 23: 679685.

[8] Seitz G, Dantonello TM, Int-Veen C, et al. Treatment efficiency outcome and surgical treatment problems in patients suffering from localized embryonal bladder/prostate rhabdomyosarcoma: a report from the cooperative soft tissue sarcoma trial CWS-96. Pediatr Blood Cancer. 2011; 56: 718-724.

[9] Stevens MC. Treatment for childhood rhabdomyosarcoma: the cost of cure. Lancet Oncol. 2005; 6: 77-84.

[10] Cadwell BT, Wilcox DT, Cost NG. Current management for pediatric urologic oncology. Adv Pediatr. 2017; 64: 191-223.

[11] Haie-Meder C, Flamant F, Revillon Y, Valayer J, Gruner M, Martelli H, et al. Place de la curiethérapie dans la stratégie thérapeutique des rhabdomyosarcomes vésicoprostatiques de l'enfant. Ann Urol 1994; 28: 302-5.

[12] Haie-Meder C, Breton-Callu C, Oberlin O, Martelli H, Audry G, Valayer J, et al. Brachytherapy in the treatment of vesicoprostatic rhabdomyosarcoma in children. Cancer Radiother. 2000; 4: 145-9.

[13] Martelli H, Haie-Meder C, Branchereau S, Franchi-Abella S, Ghigna MR, Dumas I, et al. Conservative surgery plus brachytherapy treatment for boys with prostate and/or bladder neck rhabdomyosarcoma: a single team experience. 